\title{
The Unitary Irreducible Representations of the Quantum Heisenberg Algebra
}

\author{
Alexander L. Rosenberg \\ Department of Mathematics of Harvard University, Cambridge, Massachusetts 02138, USA
}

Received March 12, 1991; in revised form June 28, 1991

\begin{abstract}
The goal of this work is to describe the irreducible representations of the quantum Heisenberg algebra and the unitary irreducible representation of one of its real forms. The solution of this problem is obtained through the investigation of the left spectrum of the quantum Heisenberg algebra using the results about spectra of generic algebras of skew differential operators (cf. [R]).
\end{abstract}

\section{Introduction}

The quantum Heisenberg algebra $H_{q, h}(k)=H_{q, h}(x, y \mid k)$ is the algebra over the field $k$ generated by indeterminables $x, y$ satisfying the relation

$$
x y-q y x=h,
$$

where $q, h \in k^{*}$. This algebra (or, rather this family of algebras) was introduced in [FG] and, it seems, is going to occupy in the quantum groups - the quantum spaces realm (cf. [Dr, J, FRT, M, S]) nearly the same place as its "classical limit" - Weyl algebra - does in representation theory (cf. [K, D]) and mathematical physics. Consequently, it deserves a thorough investigation.

The object of this work is to obtain a description of irreducible representations of the quantum Heisenberg algebra and unitary irreducible representation of one of its real forms. We come to the solution of this problem through the investigation of the left spectrum of $H_{q, h}(k)$. Recall the definition of the left spectrum.

Let $\succ$ be the following relation (preorder) on the set $I_{l} R$ of left ideals of an associative ring $R: m \succ n$ if there exists a finite subset $w \subset R$ such that the left ideal $(m: w):=\{r \in R \mid r w \subset m\}$ is contained in $n$. The left spectrum, $\operatorname{Spec}_{l} R$, of the ring $R$ consists of all the left ideals $p$ of $R$ such that $(p: x) \succ p$ for every $x \in R-p$. We use mostly the following two properties of $\left.\operatorname{Spec}_{l} R: 1\right) \operatorname{Spec}_{l} R$ contains the $\operatorname{set}^{\operatorname{Max}_{l} R}$ of maximal left ideals of $R ; 2$ ) the left spectrum is preserved by localizations (as well as left maximal ideals).

The results obtained in [R] about the left spectrum of the general algebra of skew differential operators $[A[x ; \vartheta, d]$ are sufficient to get a complete description 
of algebraically irreducible representations of the quantum Heisenberg algebra $H_{q, h}=H_{q, h}(k)$ over an arbitrary field $k$. This description is given in the first section of the article.

In the second section we obtain the list of unitary irreducible representations of the real form of the algebra $H_{q, h}(\mathbb{C})$ defined by the involution $\tau$ which sends $x$ into $y$ and $y$ into $x$.

This article is essentially a continuation of $[R]$. In particular, we use some of the notions from $[R]$ without additional explanations.

\section{The Spectrum and Irreducible Representations of the Quantum Heisenberg Algebra}

1.1. The Left Spectrum of $H_{q, h}$. Recall that $H_{q, h}$ is the algebra $A[x ; \vartheta, d]$ of $\vartheta$-skew differential operators over $A$ (cf. [R]), where $A=k[y], \vartheta(y)=q y$, and $h=d y$. We consider the case when $q$ is not a root of unity and $h(0) \neq 0$.

The left spectrum, $\operatorname{Spec}_{l} H_{q, h}$, of the algebra $H_{q, h}$ can be described as follows.

1.1.1. The "Spectral Point." There exists a "special" left maximal ideal $p_{y}=H_{q, h} y$.

The corresponding quotient module ${ }^{q} V=H_{q, h} / p_{y}$ is naturally realized as the $k$-vector space $k[x]$ of polynomials in $x$, where $x$ acts by usual multiplication.

In the case when $h \in k$, the action of $y$ is given by the formula:

$$
(x \cdot f)(x)=x f(x), \quad(y \cdot f)(x)=-(h / q) d_{q} f(x),
$$

where $d_{q}$ is the $q$-derivation:

$$
d_{q}(f)(x)=x^{-1}\left(f\left(q^{-1} x\right)-f(x)\right) /\left(q^{-1}-1\right) .
$$

In particular,

$$
y \cdot x^{n}=x^{n-1}\left(h\left(1-q^{-1}\right) /(1-q)\right.
$$

(cf. Example 3.6).

In general case, when $\operatorname{deg}(h) \geqq 1$, the action of $y$ is described by the recurrent formulas (cf. [R] Proposition 3.5).

1.1.2. Proposition. 1) For any irreducible polynomial $f=f(y)$ such that $f(0) \neq 0$, the left ideal $p_{f}:=H_{q, h} f+H_{q, h} f x$ is maximal and two-sided. The quotient ring $H_{q, h} / p_{f}$ is naturally isomorphic to the quotient field $k_{f}:=k[y] /[k[y] f$.

2) Every maximal left ideal $p$ of the algebra $H_{q, h}$ such that $p \cap k[y] \neq\{0\}$ is equivalent to one and only one of the ideals $p_{f}$, where $f=f(y)$ runs over the set of all the irreducible polynomials in $y$.

Proof. 1) Clearly, if $f(y)$ is a polynomial in $y$ such that $f(0) \neq 0$ and $\operatorname{deg}(f) \geqq 1$ then the polynomial $q_{*}^{-1} f(y)=f\left(q^{-1} y\right)$ belongs to the ideal $k[y] f$ if and only if $q=1$.

In fact, if $f\left(q^{-1} y\right)=g(y) f(y)$ then $\operatorname{deg}(g)=0$, and the equally $f(0)=q_{*}^{-1} f(0)=$ $g f(0)$ shows that $g \equiv 1$. Clearly $f\left(q^{-1} y\right)=f(y)$ only if $q=1$.

Now the statement 1) follows straightforwardly from Proposition 3.7 in [R].

2) Statement 2) is a sequence of Proposition 3.5 and Proposition 3.7 in [R]. 
1.1.3. Reflection of the Picture. In the algebra $H_{q, h}$ the variables $x$ and $y$ play a similar role. In particular, we can apply the results of Sects. 1.1.1 and 1.1.2 to the "points" $p \in \operatorname{Spec}_{l} H_{q, h}$ such that $p \cap k[x] \neq\{0\}$ and obtain the following statement:

1.1.4. Proposition. 1) The left ideal $p_{x}=H_{q, h} x$ is maximal. The corresponding simple module ${ }^{q} V_{x}=H_{q, h} / p_{x}$ is realized as the left $k[y]$-module $k[y]$ of polynomials in $y$, where (in the case when $h \in k$ ) $x$ acts as follows:

$$
(x \cdot f)(y)=h d_{q}^{\wedge} f(y)
$$

where $d_{q}^{\wedge}$ is the $1 / q$-derivation:

$$
d_{q}^{\wedge}(f)(y)=y^{-1}(f(q y)-f(y)) /(q-1) .
$$

In particular,

$$
x \cdot y^{n}=y^{n-1} h\left(1-q^{n}\right) /(1-q) .
$$

2) For any irreducible polynomial $f=f(x)$ such that $f(0) \neq 0$, the left ideal $p_{f}:=H_{q, h} f+H_{q, h} f y$ is maximal and two-sided; in particular, $p_{f}=H_{q, h} f H_{q, h}$. The quotient ring $H_{q, h} / p_{f}$ is naturally isomorphic to the quotient field $k_{f}:=k[x] / k[x] f$.

3) Every maximal left ideal $p$ of the algebra $H_{q, h}$ such that $p \cap k[x] \neq\{0\}$ is equivalent to one and only one of the ideals $p_{f}$, where $f=f(x)$ runs over the set of all the irreducible polynomials in $x$.

1.1.5. Proposition. The set of maximal left ideals $m$ of the algebra $H_{q, h}$ such that $m \cap k[y] \neq\{0\}$ and $m \cap k[x] \neq\{0\}$ consists of all the (two-sided) ideals of the form

$$
H_{q, h}(y-\lambda)+H_{q, h}\left(x-h \lambda^{-1}(1-q)^{-1}\right),
$$

where $\lambda$ runs over the set $k^{*}$ of all the invertible elements of the field $k$.

Proof. Let $\mu$ be a maximal left ideal of the algebra $H_{q, h}$ such that $\mu \cap k[y]=k[y] f$ and $\mu \cap k[x]=k[x] g$ for some nonzero polynomials $f$ and $g$.

a) Note that it is impossible that $f=y^{n}$ and $g=x^{m}$ for some integers $n \geqq 1$ and $m \geqq 1$.

Otherwise, since $y^{n-1} \notin \mu$, the ideal $\mu$ is equivalent to the ideal $H_{q, h} y$. This means, thanks to the maximality of $\mu$, that $\mu=\left(H_{q, h} y: \varphi\right)$ for a certain element $\varphi$ of the algebra $H_{q, h}$. In particular, $x^{m} \varphi \in H_{q, h} y$. But this implies immediately that the element $\varphi$ belongs to $H_{q, h} y$; hence $\mu=\left(H_{q, h} y: \varphi\right)=H_{q, h}$. Contradiction.

b) Therefore we can suppose that $f(y)=f_{1}(y) f_{2}(y)$, where $f_{1}(y)$ is an irreducible polynomial such that $f_{1}(0) \neq 0$. The ideal $\mu$ is equivalent to the ideal $\mu^{\prime}=\left(\mu: f_{2}(y)\right)$. Since $f_{1}(y) \in \mu^{\prime}, f_{1}(0) \neq 0$ and the left ideal $\mu^{\prime}$ is maximal, it is two-sided (cf. Proposition 1.1.2). This implies (since $\mu=\left(\mu^{\prime}: \psi\right)$ for some element $\psi$ from $H_{q, h} \backslash \mu^{\prime}$ ) that $\mu=\mu^{\prime}$. In particular, $f(y)=f_{1}(y)$ and $g(x)$ are irreducible polynomials, and $g(0) \neq 0$. Thus we have the equalities (cf. Proposition 1.1.2):

$$
H_{q, h} f+H_{q, h} f x=\mu=H_{q, h} g+H_{q, h} g y .
$$

In particular,

$$
f(y) x=r(x) g(x)+s(x, y) g(x) y
$$

for some polynomials $r, s$ from $H_{q, h}$. 
Replacing the left side of Eq. (1) by $x f\left(q^{-1} y\right)-q^{-1} d(f)\left(q^{-1} y\right)$ and putting $y=0$, we obtain the following:

$$
x f(0)-q^{-1} d(f)(0)=r(x) g(x) .
$$

Since $f(0) \neq 0$, Eq. (3) implies that $\operatorname{deg}(g)=1$; i.e. $g(x)=x-\alpha$ for some $\alpha \in k \backslash\{0\}$.

Now, the equality

$$
g(x) y=x y-\alpha y=q y x+h-\alpha y=q y g(x)+(q-1) \alpha y+h
$$

implies (since $g(x) y$ and $g(x)$ belong to the ideal $\mu$ ) that the element $y-(\alpha(1-q))^{-1} h$ belongs to $\mu$. Therefore $f(y)=y-(\alpha(1-q))^{-1} h$.

1.1.6. The Remaining Points: Irreducible Curves. Consider now the ideals from the left spectrum of $H_{q, h}$, which are not connected with points of Speck[y] and Spec $k[x]$; i.e. the ideals $p \in \operatorname{Spec}_{l} H_{q, h}$ such that $p \cap k[y]=\{0\}$ and $p \cap k[x]=\{0\}$.

Following the general procedure (cf. [R], 3.1, (a)), we take the localization of the algebra $H_{q, h}$ at the set of all the nonzero polynomials in $y$. The result of this localization is the algebra $H_{q, h}^{\wedge}=k(y)\left[x, q_{*}, d\right]$, which differs from $H_{q, h}=k[y]\left[x, q_{*}, d\right]$ only by rings of coefficients: the polynomial ring $k[y]$ is replaced by the field of rational functions $k(y)$.

According to the general theory, localizations are compatible with the left spectrum. In particular, the localization of the ideal $p$ at $k[y] \backslash\{0\}$ belongs to $\operatorname{Spec}_{l} H_{q, h}$. Since $H_{q, h}$ is a left and right principal ideal domain, every ideal form $\operatorname{Spec}_{l} H_{q, h}^{\hat{\lambda}}$ is equivalent to a left maximal ideal; and every left maximal ideal is of the form $H_{q, h} r$ where $r=r(x, y)$ is a polynomial in $x, y$, which is an irreducible element of the algebra $H_{q, h}$. In particular, the ideal $p$ is equal to $H_{q, h} \cap H_{q, h} r$ for some irreducible (in $H_{q, h}$ ) polynomial $r$.

Note that two polynomials, $r(x, y)$ and $r \sim(x, y)$ are equivalent, i.e. they define the same principal ideal of the algebra $H_{q, h}^{\wedge}$, if and only if $r \sim(x, y)=f(y) r(x, y)$ for some rational function $f(y)$. We can, therefore, (and will) make the choice almost unique, taking a polynomial of minimal degree in each equivalence class.

1.1.3.1. Proposition. Let a polynomial $r \in k[x, y]$ be an irreducible element of the algebra $H_{q, h}^{\hat{n}}$, having the minimal degree in its equivalence class. Then the specializations of the left ideal $\mu_{r}:=H_{q, h} \cap H_{q, h} r$ can be described as follows:

a) The ideal $\mu_{r}$ is contained in the maximal ideal $H_{q, h} f H_{q, h}=H_{q, h} f+H_{q, h} f x$ for an irreducible polynomial $f=f(y), f(0) \neq 0$, if and only if

$$
f(y / q)^{n} r\left(h d_{1 / q}(f(y)) / f(y / q), y\right) \in k[y] f,
$$

where $n$ is the degree of $r$ in $x$.

b) Similarly, the ideal $\mu_{r}$ is contained in the maximal ideal $H_{q, h} g H_{q, h}$ for an irreducible polynomial $g=g(x), g(0) \neq 0$, if and only if

$$
\left.g(q x)^{v} r\left(x,-h d_{q}(x)\right) / g(q x)\right) \in k[x] g,
$$

where $v$ is the degree of the polynomial $r$ in $y$.

c) The ideal $\mu_{r}$ is equivalent to a left maximal ideal if and only if the conditions (1) and (2) do not hold for any irreducible polynomials $f(y)$ or $g(x)$ such that $f(0) \neq 0$, $g(0) \neq 0$. 
Proof. 1) Suppose that the left ideal $\mu_{r}:=H_{q, h} \cap H_{q, h} r$ is not maximal; and let $m$ be a left maximal ideal of the ring $H_{q, h}$, containing $\mu_{r}$. Clearly $m \cap k[y] \neq\{0\}$; i.e. $m \cap k[y]=k[y] f$ for some nonzero polynomial $f=f(y)$. If $f(y)$ is not equal to $c y^{n}$ for some $c \in k$ and $n \in Z_{+}$, then $f(y)$ can be represented as $g(y) u(y)$, where $g$ is an irreducible polynomial in $y$ such that $g(0) \neq 0$. Since $u \in m$, the ideal $(m: u)$ is proper, and $\left(\mu_{r}: u\right) \subset(m: u)$. And, besides, the intersection $(m: u) \cap k[y]=k[y] g$ is a maximal ideal of $k[y]$. By Proposition 3.9 in $[\mathrm{R}]$ the ideal $(m: u)$, being left maximal, coincides with the two-sided ideal $H_{q, h} g H_{q, h}=H_{q, h} g+H_{q, h} g x$. This implies that $m=(m: u)=H_{q, h} g+H_{q, h} g x=k[y] g+H_{q, h}(x-\psi)$, where $\psi=\psi(y)$ is a polynomial in $y$ such that $\psi(y) g(y / q)=d(g(y / q))+f(y) g(y)$ for some $f(y) \in k[y]$ (cf. the proof of Proposition 3.7 in [R]).

The ideal $m=k[y] g+H_{q, h}(x-\psi)$ consists of all the "polynomials" $f(x, y)$ such that $f(\psi(y), y) \in k[y] g$. In particular, $r(\psi(y), y) \in k[y]$. On the other hand, it is easy to see that, thanks to the minimality of $\operatorname{deg}(r), \mu_{r} \in m$ if $r(\psi(y), y) \in k[y] g$.

Note that the last inclusion is equivalent to the following one:

$$
g(y / q)^{n} r(d(g(y / q)) / g(y / q), y) \in k[y] g,
$$

where $n$ is the degree of $r$ as a polynomial in $x$.

Proposition 1.1.3.1 finishes the description of the left spectrum of the quantum Heisenberg algebra $H_{q, h}=H_{q, h}(k)$ over an arbitrary field $k$. It is convenient, however, to have a separate picture in the case of an algebraically closed field $k$.

1.2. The Left Spectrum of the Quantum Heisenberg Algebra Over an Algebraically Closed Field. Let the field $k$ be algebraically closed. Then we have

1) Closed points:

(a) the ideals $H_{q, h} x$ and $H_{q, h} y$;

(b) the two-sided (left and right) maximal ideals

$$
m_{\lambda}=H_{q, h}(y-\lambda)+H_{q, h}\left(x-h \lambda^{-1}(1-q)^{-1}\right),
$$

where $\lambda$ runs over the set $k^{*}$ of nonzero elements of the field $k$;

(c) the ideals of the form $\mu_{r}=H_{q, h} \cap H_{q, h} r$, where the polynomial $r=r(x, y) \in$ $k[y][x]$ is an irreducible element of the algebra $H_{\dot{q}, h}$ such that

$$
r\left(h \lambda^{-1}(1-q)^{-1}, \lambda\right) \neq 0 \text { for each } \lambda \in k^{*} \text {. }
$$

The condition (1) guarantees that the left ideal $\mu_{r}$ is maximal and has zero intersections with $k[x]$ and $k[y]$.

Every maximal left ideal of the algebra $H_{q, h}$ having a nonzero intersection with $k[y]$ or $k[x]$ coincides with one and only one of the ideals from (a) and (b). The ideals $H_{q, h} x, H_{q, h} y$ and the ideals $m_{\lambda}$ for different $\lambda \in k^{*}$ are pairwise non-equivalent.

Every maximal left ideal of the algebra $H_{q, h}$, which has a trivial intersection with the subalgebras $k[x]$ and $k[y]$, coincides with one of the ideals $\mu_{r}$ with $r$ satisfying condition (1).

2) Non-closed points:

(d) the generic point $\{0\}$;

(e) the ideals $H_{q, h}(y-\lambda)$, where $\lambda$ runs over the set $k^{*}$ of nonzero elements of the field $k$;

(e^) the ideals $H_{q, h}(x-\eta)$, where $\eta$ runs over the set $k^{*}$;

(f) the ideals $\mu_{r}=H_{q, h} \cap H_{\hat{q}, h} r$, where the polynomial $r=r(x, y) \in k[y][x]$ is an 
irreducible element of the algebra $H_{q, h}$ such that

$$
\operatorname{deg}(r) \leqq \operatorname{deg}(u) \quad \text { for any } u \in \mu_{r}
$$

and the equation

$$
r\left(h \lambda^{-1}(1-q)^{-1}, \lambda\right)=0
$$

has solutions in $k^{*}$.

Each of the ideals $H_{q, h}(y-\lambda)$, and $H_{q, h}(x-\eta), \eta, \lambda \in k^{*}$, has only one specialization, which is the maximal (two-sided) ideal $m_{\lambda}=H_{q, h}(y-\lambda) H_{q, h}$ and $m_{\eta} \hat{\imath}:=H_{q, h}(x-\eta) H_{q, h}$ respectively. We can write it down as follows:

$$
\begin{array}{cc}
H_{q, h}(y-\lambda) H_{q, h}=H_{q, h}\left(x-\lambda^{\wedge}\right) H_{q, h} \\
\nearrow & \nwarrow \\
H_{q, h}(y-\lambda) & H_{q, h}\left(x-\lambda^{\wedge}\right)
\end{array}
$$

where the ("conjugate" to $\lambda$ ) element $\lambda^{\wedge}$ is equal to $h \lambda^{-1}(1-q)^{-1}$.

Every (non-trivial) specialization of the ideal $\mu_{r}=H_{q, h} \cap H_{q, h}^{\hat{\lambda}} r$ (cf. the point (b) above) is a maximal two-sided ideal $H_{q, h}\left(y-\lambda^{\prime}\right) H_{q, h}$ for some $\lambda^{\prime} \in k^{*}$. Solutions $\lambda \in k^{*}$ of Eq. (3) give all the specializations of the ideal $\mu_{r}$ in the "strong" sense: $\mu_{r} \subset H_{q, h}(y-\lambda) H_{q, h}$.

It seems, however, more than probable that the set of the solutions of Eq. (3) for some equivalent to the $\mu_{r}$ ideal, say $\left(\mu^{r}:(y-\lambda)\right)$ for a certain $\lambda$ such that $\mu_{r} \subset H_{q, h}(y-\lambda) H_{q, h}$, can not coincide with the set of solutions for $r$.

\section{In Search of Unitary Representations}

2.1. Definition. Let $A$ be an algebra over the field $\mathbb{C}$ of complex numbers. $A$ map $\sigma: A \rightarrow A$ is called an involution if

$$
\sigma(x y)=\sigma(y) \sigma(x) \text { and } \sigma(\lambda x)=\bar{\lambda} \sigma(x)
$$

for any $x, y \in A$ and $\lambda \in \mathbb{C}$.

2.2. Involutions of the Quantum Heisenberg Algebra. From now on $k$ will be the field of complex numbers.

2.2.1. Lemma. The involutions of the quantum Heisenberg algebra $H_{q, h}$ are classified as follows.

(i) Let $q$ and $h$ be real numbers. Then the maps

$$
\tau_{b}: x \mapsto b y, \quad y \mapsto b^{-1} x,
$$

where $b$ runs over the set $k^{*}$ of nonzero complex numbers, determine all the involutions of the algebra $H_{q, h}$.

(ii) Let $|q|=1$ and $h=i q^{1 / 2} \lambda$ for some real number $\lambda$. Then there are only two involutions, $\tau_{+}$and $\tau_{-}$, given by the formulas:

$$
\tau_{+}(x)=x, \quad \tau_{+}(y)=y \quad \text { and } \quad \tau_{-}(x)=-x, \quad \tau_{-}(y)=-y .
$$


(iii) Let $|q|=1$ and $h=q^{1 / 2} \lambda$ for some real number $\lambda$. Then there are only two involutions, $\tau_{+}$and $\tau_{-}$, defined as follows:

$$
\tau_{\hat{+}}(x)=x, \quad \tau_{+}(y)=-y \quad \text { and } \quad \hat{\tau_{-}}(x)=-x, \quad \tau_{-}(y)=y .
$$

(iv) There are no involutions for other pairs $(\lambda, h)$.

Proof. Let $\sigma(x)=a x+b y$ and $\sigma(y)=c x+d y$. The relations, which should hold are:

$$
\begin{aligned}
\bar{h} & =\sigma(x y-q y x)=\sigma(y) \sigma(x)-\bar{q} \sigma(x) \sigma(y)=(c x+d y)(a x+b y)-\bar{q}(a x+b y)(c x+d y) \\
& =c a(1-\bar{q}) x^{2}+c b x y+d a y x+d b(1-\bar{q}) y^{2}-\bar{q} a d x y-\bar{q} b c y x \\
& =d b(1-\bar{q}) y^{2}+(b c(q-\bar{q})+a d(1-q \tilde{q})) y x+c a(1-\bar{q}) x^{2}+(c b-\bar{q} a d) h .
\end{aligned}
$$

Thus we obtain the following equations:

$$
\begin{gathered}
b d(1-\bar{q})=0, \quad a c(1-\bar{q})=0, \\
(b c(q-\bar{q})+a d(1-q \bar{q}))=0, \quad(c b-\bar{q} a d) h=\bar{h} .
\end{gathered}
$$

Since $q \neq 1$, the first two equations are equivalent to

$$
b d=0, \quad a c=0 .
$$

Therefore we have the following posssibilities:
1) $b=a=0$;
2) $d=a=0$;
3) $b=c=0$;
4) $d=c=0$.

That is all, because if any three of the elements $a, b, c, d$ are equal to zero, then, as it follows from the last of Eqs. (2), $\bar{h}=0$. The solutions $b=a=0$ and $c=d=0$ are also incompatible with this equation. Consider the remaining possibilities (2).

2) $a=d=0$. Then

$$
b c(q-\bar{q})=0, \quad b c h=\bar{h} .
$$

The property $\sigma^{2}=i d$ is equivalent to the equality $b c=1$. Therefore the equalities (4) mean exactly that $q$ and $h$ are real numbers.

3) $b=c=0$. Then the property $\sigma^{2}=i d$ and (2) provide the following equations:

$$
a^{2}=d^{2}=1, \quad a d(1-q \bar{q})=0, \quad-\bar{q} a d h=\bar{h} .
$$

Since $a d \neq 0$, the second Eq. (5) means exactly that $|q|=1$. It follows from the first and last Eq. (5) that $h= \pm q \bar{h}$.

(i) $a=d= \pm 1$. Then $h=-q \bar{h}$. This equality means that $h=i q^{1 / 2} \lambda$ for some real number $\lambda$. $\lambda$.

(ii) $a=-d= \pm 1$. Then $h=g \bar{h}$; or, equivalently, $h=q^{1 / 2} \lambda$ for some real number

\subsection{Unitary Representations of the Quantum Heisenberg Algebra}

2.3.1. Definition. Let $R$ be an algebra with the involution *. A representation $\rho$ of the algebra $R$ in the complex vector space $V$ is referred to as unitary, or *-unitary, 
if there exists a positive definite Hermitian inner product (,) such that

$$
(\rho(x) v \cdot w)=\left(v \cdot \rho\left(x^{*}\right) w\right)
$$

for any two vectors $v, w \in V$ and for any element $x \in R$.

Our goal is to describe the unitary representations of the algebra $H_{q, h}$ (if any), corresponding to different involutions of $H_{q, h}$ (cf. Lemma 2.2.1).

2.3.2. Proposition. Let $h$ and $q$ be real numbers. Then

a) The "standard" representation of the algebra $H_{q, h}$ in the vector space $k[z]$ of polynomials in $z$,

$$
(x \cdot f)(z)=z f(z), \quad(y \cdot f)(z)=-(h / q) \partial_{1 / q} f(z)
$$

(cf. 4.1.1) has the unique (up to multiplication by a positive number) $\tau_{b}$-unitary structure, where $\tau_{b}$ is an arbitrary involution of the algebra $H_{q, h}$ :

$$
\tau_{b}: x \mapsto b y, \quad y \mapsto b^{-1} x,
$$

if and only if $h / q<0$.

b) The "standard" representation of the algebra $H_{q, h}$ in the vector space $k[z]$ of polynomials in $z$,

$$
(y \cdot f)(z)=z f(z), \quad(x \cdot f)(z)=h \partial_{q} f(z)
$$

has the unique (up to the multiplication by a positive number) $\tau_{b}$-unitary structure if and only if $h>0$.

Proof. a) Let $v_{i}=z^{i}, i \geqq 0$. It is easy to see that a $\tau_{b}$-invariant inner product (if any) satisfies the equalities:

$$
\left(v_{i}, v_{i}\right)=h\left(1-q^{-i}\right) /(1-q)\left(v_{i-1}, v_{i-1}\right) .
$$

This implies immediately that $\left(v_{i}, v_{i}\right)$ is positive for every $i \geqq 0$ if and only if $\left(v_{0}, v_{0}\right)>0$ and $h / q<0$. Thus, if $h / q<0$ then there exists a unique inner product (,) on ${ }^{q} V$ such that $(1,1)=1$ and the representation ${ }^{q} V$ is unitary. The elements $(h / q)^{-i / 2}([-i] !)^{-1 / 2} x^{i}, i \geqq 0$, form an orthonormal basis of ${ }^{q} V$ with respect to $($,$) .$

b) Similarly, in case of the canonical representation

$$
(y \cdot f)(z)=z f(z), \quad(x \cdot f)(z)=h \partial_{q} f(z),
$$

it follows from the recurrent relations

$$
\left(v_{i}, v_{i}\right)=h\left(1-q^{i}\right) /(1-q)\left(v_{i-1}, v_{i-1}\right)
$$

that the inequality $h>0$ is necessary and sufficient for the existence of $\tau_{b}$-invariant positive Hermitian form of $k[z]$.

2.3.3. Proposition. Let $k$ be a field of complex numbers, $q \neq 1$ and $h$ real numbers and $H_{q, h}=H_{q, h}(k)$ the corresponding quantum Heisenberg algebra over the field $k$. And let $\tau_{b}$ be the involution $x \mapsto b y, y \mapsto b^{-1} x, b \in k$. Then there are the following possibilities:

1) If $q>0$ and $h b^{-1}(1-q)^{-1}>0$, then each point $\gamma$ of the circle of the radius $h b^{-1}(1-q)^{-1}$ determines a one-dimensional $\tau_{b}$-unitary representation

$$
\chi_{\gamma}: x \mapsto h r^{-1}(1-q)^{-1}, \quad y \mapsto \gamma .
$$


2) If $q<0$ and $h b(1-q)^{-1}>0$, then

(a) Each point $\gamma$ of the circle of the radius $h b^{-1}(1-q)^{-1}$ determines a one-dimensional $\tau_{b}$-unitary representation

$$
\chi_{\gamma}: x \mapsto h \gamma^{-1}(1-q)^{-1}, \quad y \mapsto \gamma .
$$

(b) Each point $\lambda$ of the open interval $\left(0, h b(1-q)^{-1}\right)$ determines an algebraically irreducible infinite dimensional $\tau_{b}$-unitary representation of $H_{q, h}$.

3) If $b$ is real (as it is in the previous cases), then the remaining $\tau_{b}$-unitary irreducible representations contain the "standard" representation,

$$
(y \cdot f)(z)=z f(z), \quad(x \cdot f)(z)=h \partial_{q} f(z) .
$$

This is the complete (up to equivalence) list of irreducible $\tau_{b}$-unitary representations of $H_{q, h}$.

Proof. Let $\rho$ be an irreducible $\tau_{b}$-unitary representation of the algebra $H_{q, h}$. Denote by $L$ the operator $b \rho(y x)$. Since $b y x=\tau_{b}(x) x$, and, by condition, $\rho\left(\tau_{b}(x)\right)=\rho(x)^{*}$, the operator $L$ is positive.

Let $v$ be an eigenvector of the operator $L$ with the eigenvalue $\lambda$. Then it follows from the relations

$$
y x y=y(q y x+h), \quad y x x=q^{-1}(x y-h) x=q^{-1} x(y x-h)
$$

that

$$
\begin{gathered}
L \rho(y) v=b \rho(y x y) v=\rho(y)(q L+b h) v=\rho(y)(q \lambda+b h)=(q \lambda+b h)(\rho(y) v) \\
L \rho(x)=q^{-1} \rho(x)(L-b h) v=q^{-1} \rho(x)(\lambda-b h) v=q^{-1}(\lambda-b h)(\rho(x) v) .
\end{gathered}
$$

In other words, $\rho(y) v$ is the eigenvector of the operator $L$ with the eigenvalue $(q \lambda+b h)$, and $\rho(x) v$ is the eigenvector of the operator $L$ with the eigenvalue $q^{-1}(\lambda-b h)$. Note that, since all the eigenvalues of the operator $L$ are non-negative (in particular, real) numbers, this implies that $b$ should be a real (nonzero) number.

Let now $\lambda$ be the minimal positive eigenvalue of the operator $L$ (since $L$ is positive, then existence of any eigenvalue guarantees the existence of the minimal one). And suppose that both vectors, $\rho(y) v$ and $\rho(x) v$, are non-zero. Then the inequalities

$$
(q \lambda+b h) \geqq \lambda, \quad q^{-1}(\lambda-b h) \geqq \lambda
$$

hold. Consider each to the two possibilities: $q>0$ and $q<0$.

1) $q>0$. Then the inequalities (1) can be rewritten in the form $(q-1) \lambda+b h \geqq 0$, $(q-1) \lambda+b h \leqq 0$. Therefore (since $q \neq 1)$ we have:

$$
\lambda=b h(1-q)^{-1}>0 \text {. }
$$

The annihilator of the vector $v$ contains the left ideal of the algebra $H_{q, h}$ generated by the element $y x-h(1-q)^{-1}$.

Note now that, for an arbitrary $\gamma \in k$, we have the identity:

$$
y x-h(1-q)^{-1}=(y-\gamma) x+\gamma\left(x-h \gamma^{-1}(1-q)^{-1}\right) .
$$

The identity (3) means that the left ideal $H_{q, h}\left(y x-h(1-q)^{-1}\right)$ is contained in the (two-sided) maximal ideal $m_{\gamma}:=H_{q, h}(y-\gamma)+H_{q, h}\left(x-h \gamma^{-1}(1-q)^{-1}\right)$ for every $\gamma \in k$ (cf. 1.2). Since, by hypothesis, the representation $\rho$ we are considering is 
irreducible, it is equivalent to the one-dimensional representation

$$
\chi_{\gamma}: x \mapsto h \gamma^{-1}(1-q)^{-1}, \quad y \mapsto \gamma
$$

for some $\gamma \in k$. The requirement of $\tau_{b}$-unitarity of the representation $\chi_{\gamma}$ means exactly that

$$
\begin{gathered}
b \gamma=\chi_{\gamma}(b y)=\chi_{\gamma}\left(\tau_{b}(x)\right)=\overline{\chi_{\gamma}(x)}=h(1-q)^{-1} / \bar{\gamma} \\
h(b \gamma)^{-1}(1-q)^{-1}=\chi_{\gamma}(x / b)=\chi_{\gamma}\left(\tau_{b}(y)\right)=\overline{\chi_{\gamma}(y)}=\bar{\gamma} .
\end{gathered}
$$

Or, equivalently,

$$
|\gamma|^{2}=h b^{-1}(1-q)^{-1} \text {. }
$$

Thus each point of the circle of the radius $h b^{-1}(1-q)^{-1}$ determines a onedimensional $\tau_{b}$-unitary representation.

2) $q<0$. Then the inequalities (1) are equivalent one to another. From the first of them we obtain:

$$
0<\lambda \leqq b h(1-q)^{-1} .
$$

Take an arbitrary $\lambda$ satisfying (3). The element $y x-\lambda / b$, which annihilates the vector $v$, can be represented in the form

$$
y x-\lambda / b=(y-\gamma) x+\gamma\left(x-h \gamma^{-1}(1-q)^{-1}\right)+h(1-q)^{-1}-\lambda / b .
$$

This shows that the element $y x-\lambda / b$ belongs to the maximal ideal $m_{\gamma}$ for some $\gamma$ if and only if

$$
\lambda=b h(1-q)^{-1} .
$$

I.e. we are in the situation described in the Sect. 1) of the proof. Using its result, we obtain: each point of the circle of the radius $h b^{-1}(1-q)^{-1}$ determines a onedimensional $\tau_{b}$-unitary representation.

If, on the contrary, $\lambda<b h(1-q)^{-1}$, then the left ideal generated by $y x-\lambda / b$ is maximal and, therefore, determines an (algebraically) irreducible infinitedimensional $\tau_{b}$-unitary representation.

Now we describe the rest of $\tau_{b}$-unitary representation; i.e. the representations, for which the condition

$$
(q \lambda+b h) \geqq \lambda, \quad q^{-1}(\lambda-b h) \geqq \lambda
$$

does not hold.

3) Let $q>0$.

3.1) Suppose that the first of the inequalities (1) is in fault; i.e.

$$
\lambda(1-q)>b h .
$$

This means that the operator $\rho(y)$ annihilates the vector $v$; and, therefore there is an embedding (with a dense image) of the standard representation ${ }^{q} V$ into the representation $\rho$. In particular, ${ }^{q} V$ is a $\tau_{b}$-unitary representation. According to Proposition 2.3.2, this implies that $h / q<0$; i.e., since by condition $q>0, h<0$.

3.2) The second possibility,

$$
\lambda(1-q)<b h
$$


means that the operator $\rho(x)$ annihilates the vector $v$; i.e. $\rho$ contains the "standard" representation

$$
(y \cdot f)(z)=z f(z), \quad(x \cdot f)(z)=h \partial_{q} f(z) .
$$

It is possible if and only if $h>0$.

4) Consider now the case $q<0$. Then the inequality (6) implies that $q<-1$, and (7) implies that $q>-1$ (cf. Proposition 2.3.2). Therefore, again, as in the case $q>0$, no more than one of the two standard representations can be $\tau_{b}$-unitary.

Acknowledgement. I am grateful to David Kazhdan for inspiring discussions in connection with this work.

\section{References}

[D] Dixmier, J.: Algebras Enveloppantes. Paris, Bruxelles, Montreal: Gauthier-Villars 1974

[Dr] Drinfeld, V. G.: Quantum Groups, Proc. Int. Cong. Math. pp. 798-820. Berkeley 1986

[FG] Gelfand, I. M., Fairline, D. B.: The Algebra of Weyl Symmetrized Polynomials and its Quantum Extension. Commun. Math. Phys. 13 (1991)

[FRT] Faddeev, L., Reshetikhin, N., Takhtajan, L.: Quantization of Lie Groups and Lie Algebras, preprint, LOMI-15-87; Algebra Analysis, vol 1, (1) (1989)

[J] Jimbo, M.: A $q$-Difference Analog of $U(g)$ and the Yang-Baxter Equation. Lett. Math. Phys. 10, 63-69 (1985)

[K] Kirillov, A.: Unitary representations of Unipotent Groups. Russ Math. Survey 17, 57-110 (1962)

[M] Manin, Yu. I.: Quantum Groups and Non-commutative Geometry. CRM, Université de Montréal (1988)

[R] Rosenberg, A. L.: The Spectrum of the Algebra of Skew Differential Operators and the Irreducible Representations of the Quantum Heisenberg Algebra. Commun. Math. Phys. 142, 567-588 (1991)

[R1] Rosenberg, A. L.: Noncommutative Affine Semischemes and Schemes, "Seminar on Supermanifolds", vol 26, pp 1-317. Reports of Dept. of Math. of Stockholm Univ. 1988

[R2] Rosenberg, A. L.: Left Spectrum, Levitzki Radical and Noncommutative Schemes. Proc. Natl. Acad. 87 (1990)

[S] Smith, S. P.: Quantum Groups: an Introduction and Survey for Ring Theorists, preprint

Communicated by N. Yu. Reshetikhin 
